\title{
Informing neutron capture nucleosynthesis on short-lived nuclei with $(d, p)$ reactions
}

\author{
Jolie A. Cizewski ${ }^{1}{ }^{*}$, Andrew Ratkiewicz ${ }^{1,2}$, Jutta E. Escher ${ }^{2}$, Alexandre Lepailleur ${ }^{1}$, \\ Steven D. Pain ${ }^{3}$, and Gregory Potel $^{4}$ for the STARLiTeR and GODDESS collaborations \\ ${ }^{1}$ Department of Physics and Astronomy, Rutgers University, New Brunswick, NJ 08901 USA \\ ${ }^{2}$ Nuclear \& Chemical Sciences Division, Lawrence Livermore National Laboratory, CA, 94550 USA \\ ${ }^{3}$ Physics Division, Oak Ridge National Laboratory, Oak Ridge, TN 37831 USA \\ ${ }^{4}$ Department of Physics \& Astronomy, Michigan State University and the Facility for Rare Isotope \\ Beams, East Lansing, MI 48824 USA
}

\begin{abstract}
Neutron capture on unstable nuclei is important in understanding abundances in r-process nucleosynthesis. Previously, the non-elastic breakup of the deuteron in the $(\mathrm{d}, \mathrm{p})$ reaction has been shown to provide a neutron that can be captured by the nucleus and the gamma-ray decay of the subsequent compound nucleus can be modelled to predict the gamma-ray decay of the compound nucleus in the $(n, \gamma)$ reaction. Preliminary results from the ${ }^{95} \mathrm{Mo}(\mathrm{d}, \mathrm{p} \gamma)$ reaction in normal kinematics support the $(\mathrm{d}, \mathrm{p} \gamma)$ reaction as a valid surrogate for neutron capture. The techniques to measure the $(\mathrm{d}, \mathrm{p} \gamma)$ reaction in inverse kinematics have been developed.
\end{abstract}

\section{Introduction}

Neutron capture reactions are responsible for the synthesis in stars of essentially all of the elements heavier than iron through either the $s$ or $r$ process. While the $s$ process proceeds near stable nuclei, the $r$-process waiting points are short-lived and far from stability. Recent studies [1] have demonstrated that unknown $(n, \gamma)$ rates on nuclei near the $r$-process path, and in particular near closed neutron shells, could have significant impact on predicting abundances with $r$-process network calculations. Constraining (n, $\gamma)$ rates could also serve to inform our knowledge of the site of the $r$ process.

Neutron capture near closed shells can proceed by two processes. Direct (including semi-direct) capture can be deduced if the spectroscopic factors of low-spin states have been measured, for example, with $(\mathrm{d}, \mathrm{p})$ reactions with radioactive ${ }^{126,128,130,132} \mathrm{Sn}$ beams [2]. For open neutron shell nuclei, neutron capture is expected to proceed predominantly through the population of a compound nucleus with gamma decay that proceeds by many paths. While the population of the compound nucleus can be calculated with optical models, the decay depends upon the level density and $\gamma$-ray strength function, properties that cannot be accurately extrapolated to weakly bound nuclei, far from stability. Because

* Corresponding author : cizewski@physics.rutgers.edu 
of the importance of $(n, \gamma)$ cross sections on short-lived neutron-rich nuclei in understanding r-process nucleosynthesis, and also in applications of nuclear physics, it is important to validate a surrogate for neutron capture and in particular to inform the decay of compound nuclei [3].

The $(\mathrm{d}, \mathrm{p} \gamma)$ reaction is a promising surrogate for $(\mathrm{n}, \gamma)$ and for measurements with radioactive ion beams (RIBs). Potel and collaborators have recently developed the framework [4] in which the non-elastic breakup of the deuteron provides a neutron that can be captured by the nucleus. This framework also provides an angular momentum decomposition as a function of excitation energy (effective neutron energy) as well as the fraction of the $(d, p)$ reaction cross section in the elastic breakup channel. Escher and colleagues [5] have developed the techniques to fit surrogate reaction gamma-ray decay probabilities as a function of excitation energy to extract the Hauser-Feshbach (HF) decay of the compound nucleus. The decay of the compound nucleus is corrected for the differences in entry angular momentum between the $(\mathrm{d}, \mathrm{p})$ reaction (predicted within the Potel framework) and neutron capture. This corrected decay of the compound nucleus is combined with the cross section for the target + neutron system calculated with optical models to predict $(n, \gamma)$ cross sections as a function of neutron energy.

The $(\mathrm{d}, \mathrm{p})$ reaction can also be used to deduce the direct-semi-direct component of neutron capture as a function of neutron energy important in nuclei with low neutron separation energies $\left(S_{n}\right)$, for example near closed neutron shells. Finally the $(d, p)$ reaction is well suited for measurements with RIBs in inverse kinematics, where events measured at angles $>90^{\circ}$ in the laboratory are dominated by reaction protons, with other channels, such as elastic and inelastic scattering, observed at angles forward of $90^{\circ}$.

\section{Validating (d,p $\gamma)$ as $(n, \gamma)$ surrogate}

The ${ }^{95} \mathrm{Mo}+$ neutron system [6] was chosen to validate the (d,p $\left.\gamma\right)$ reaction as a neutron capture surrogate because the $(\mathrm{n}, \gamma)$ cross section has been measured up to $200 \mathrm{keV}[7,8]$. Also, the final nucleus ${ }^{96} \mathrm{Mo}$ is even-even, with a relatively simple decay scheme at low excitation energies, and the level scheme is well-established [8]. The primary validation experiment was conducted at the Texas A\&M Cyclotron Institute with $\approx 12.5-\mathrm{MeV}$ deuteron beams interacting with an enriched ${ }^{95} \mathrm{Mo}$ target. The reaction charged particles and gamma rays were measured in singles and coincidence with the STAR-LiTeR system of annular silicon strip and Compton-suppressed high-purity clover Ge detectors [9]. A preliminary gamma-ray energy as a function of excitation energy (measured with protons) matrix is displayed in Figure 1. Below the neutron separation energy $S_{n}$, all of the gamma rays are in ${ }^{96} \mathrm{Mo}$. Above $S_{n}$, surrogate neutron capture (transitions in ${ }^{96} \mathrm{Mo}$ ) and surrogate $\left(n, n^{\prime}\right)$ transitions in ${ }^{95} \mathrm{Mo}$ are observed. By gating on excitation energy, the gamma-ray emission probability as a function of excitation energy (or effective neutron energy) can be deduced, as displayed in Figure 2. The $778-\mathrm{keV} 2^{+} \rightarrow 0^{+}$transition in ${ }^{96} \mathrm{Mo}$ collects essentially all of the gamma-ray intensity with very little intensity bypassing the $2^{+}$state, e.g., via the $2_{2}{ }^{+} \rightarrow 0^{+}$transition. Also the $778-\mathrm{keV}$ transition is about a factor of five stronger than the $850-\mathrm{keV} 4^{+} \rightarrow 2^{+}$yrast transition, with the $812-\mathrm{keV} 6^{+} \rightarrow 4^{+}$yrast transition a factor of 20 lower. This supports a relatively low angular momentum transfer even at high excitation energies approaching $\mathrm{S}_{\mathrm{n}}$.

Preliminary results for fitting the measured gamma-ray intensity probabilities are displayed in Figure 2. These account for the angular momentum transferred in the surrogate reaction. The decay model resulting from the fit was combined with optical model predictions for the formation of the ${ }^{95} \mathrm{Mo}+\mathrm{n}$ compound nucleus to deduce the surrogate 


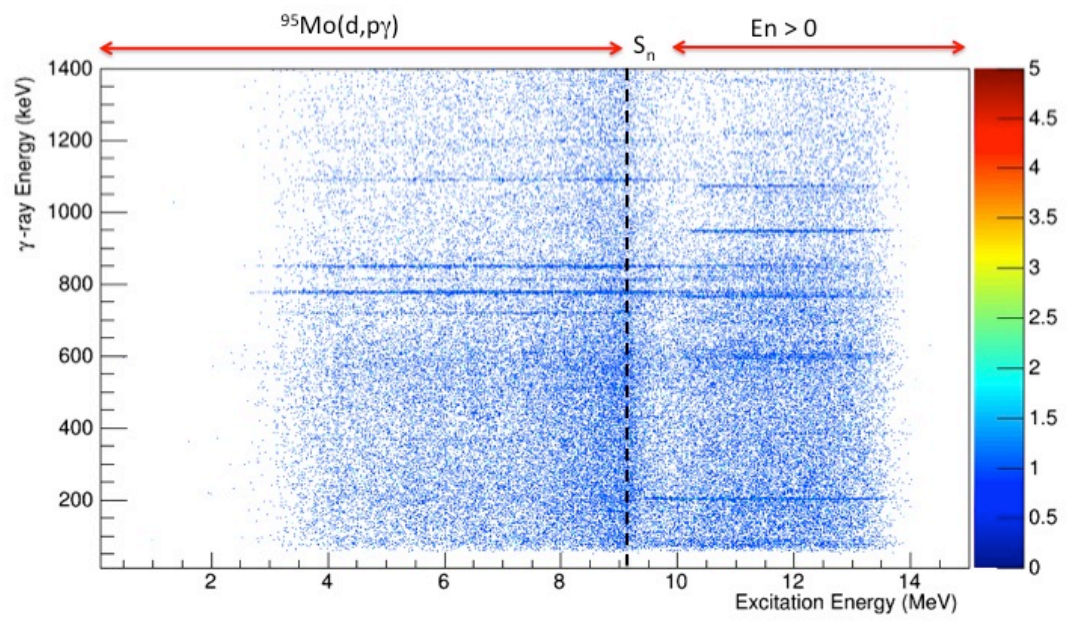

Figure 1. Preliminary gamma-ray energy as a function of excitation energy (measured with protons) matrix for the ${ }^{95} \mathrm{Mo}(\mathrm{d}, \mathrm{p} \gamma)$ reaction. The vertical line represents neutron separation energy $\mathrm{S}_{\mathrm{n}}$ that is also the zero for effective neutron energies.

$(\mathrm{n}, \gamma)$ cross sections. Preliminary results indicate good agreement between measured, evaluated and surrogate-reaction deduced $(n, \gamma)$ cross sections. Next steps include incorporating uncertainties in the modelling of the ${ }^{95} \mathrm{Mo}+\mathrm{d}$ system.

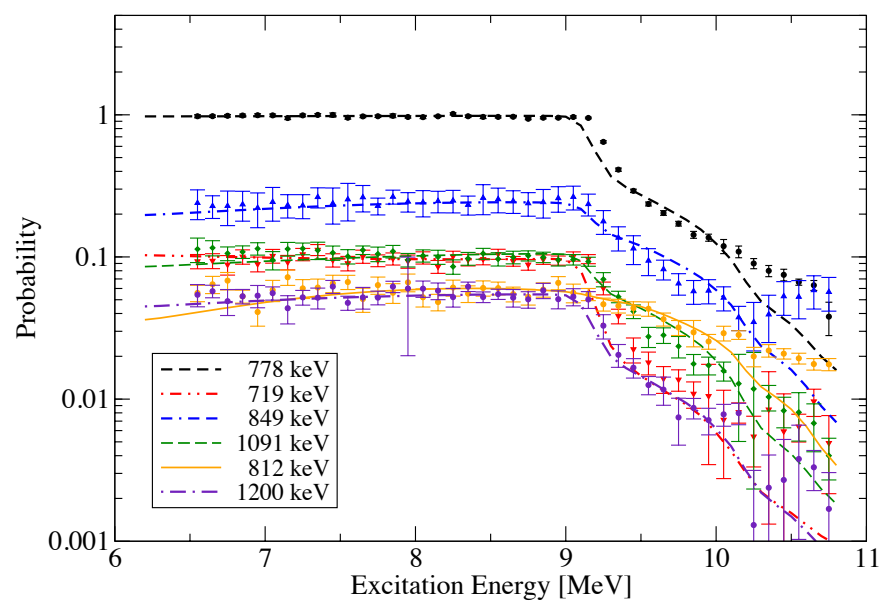

Figure 2. Gamma-ray emission probability as a function of excitation energy for transitions in ${ }^{96} \mathrm{Mo}$. The dashed lines represent the HF preliminary predictions of the $\gamma$-ray emission probabilities.

\section{Developing techniques to measure (d,py) reactions with RIBs}

We have developed the capabilities to measure the $(\mathrm{d}, \mathrm{p} \gamma)$ reaction in inverse kinematics on the path to realizing such measurements with RIBs interacting with $\mathrm{CD}_{2}$ targets. Light reaction products are detected in the Oak Ridge Rutgers University Barrel Array (ORRUBA) of position-sensitive silicon-strip detectors augmented by annular arrays of graduated silicon-strip detectors at back and forward angles in the laboratory. Reaction gamma rays are detected in the Gammasphere array of Compton-suppressed Ge detectors to realize the GODDESS system [10]: Gammasphere ORRUBA Dual Detectors for 
Experimental Structure Studies. As a result of analyzing the particle-gamma coincidences, new states at $\mathrm{E}_{\mathrm{x}} \approx 2 \mathrm{MeV}$ have been identified as candidates for $2 \mathrm{f}_{7 / 2}$ and $3 \mathrm{p}_{3 / 2}$ excitations above the $\mathrm{N}=82$ shell gap. Above the neutron separation energy, transitions in the (n,n') surrogate ${ }^{134} \mathrm{Xe}$ nucleus are observed. Further analysis of the $(\mathrm{d}, \mathrm{p} \gamma)$ reactions with ${ }^{134} \mathrm{Xe}$ and ${ }^{95}$ Mo beams (to demonstrate a surrogate in inverse kinematics) are in progress.

\section{Summary and conclusions}

In summary, we are demonstrating that the $(d, p \gamma)$ reaction is a valid surrogate for the $(n, \gamma)$ reaction important in understanding observed abundances in $r$-process nucleosynthesis. The Potel theoretical framework has shown how non-elastic breakup of the deuteron in the $(\mathrm{d}, \mathrm{p})$ reaction provides a neutron to be captured. Level density and gamma-ray strength functions from $(\mathrm{d}, \mathrm{p} \gamma)$ reactions can be deduced and used in calculating $(\mathrm{n}, \gamma)$ cross sections. The techniques to measure $(\mathrm{d}, \mathrm{p} \gamma)$ reactions with RIBs have been developed. First experiments with $\mathrm{N} \approx 82$ nuclei near the $r$-process path of nucleosynthesis are being planned.

This work is supported in part by the U.S. Department of Energy National Nuclear Security Administration under the Stewardship Science Academic Alliances program DE-FG52-09NA29467 and DE-NA0000979, LLNL DE-AC52-07NA27344 and LDRD 16-ERD-022 and the Office of Nuclear Physics ORNL DE-AC05-00OR22725 and the National Science Foundation.

\section{References}

1. M. Mumpower et al., Prog. Nucl. Part. Phys. 86, 86 (2016)

2. R. L. Kozub et al., Phys. Rev. Lett. 109, 172501 (2012) and B. Manning et al., (submitted 2017) and references therein.

3. Almudena Arcones et al., Prog. Nucl. Part. Phys. 94, 1 (2017)

4. G. Potel, F. M. Nunes, and I. J. Thompson, Phys. Rev. C 92, 034611 (2015)

5. J. E. Escher et al., Rev. Mod. Phys. 84, 353 (2012) and J. E. Escher et al., EPJ Web of Conferences 122, 12001 (2016)

6. A. Ratkiewicz et al., EPJ Web of Conferences 93, 02012 (2015)

7. A. De L. Musgrove et al., Nucl. Phys. A 270, 108 (1976)

8. ENDF and ENSDF databases http://www.nndc.bnl.gov

9. S. Lesher et al., Nucl. Inst. \& Meth. in Phys. Res. A 621, 286 (2010) and

R. J. Casperson et al., Phys. Rev. C 90, 034601 (2014)

10. S.D. Pain, et al., Physics Procedia (in press, 2017) 